TAIWANESE JOURNAL OF MATHEMATICS

Vol. 11, No. 1, pp. 43-50, March 2007

This paper is available online at http://www.math.nthu.edu.tw/tjm/

\title{
NORMALIZED MATCHING PROPERTY OF A CLASS OF SUBSPACE LATTICES
}

\author{
Jun Wang and Huajun Zhang*
}

\begin{abstract}
Let $V_{n}(q)$ be the $n$-dimensional vector space over the finite field with $q$ elements and $K$ a selected $k$-dimensional subspace of $V_{n}(q)$. Let $C[n, k, t]$ denote the set of all subspaces $S$ 's such that $\operatorname{dim}(S \cap K) \geq t$. We show that $C[n, k, t]$ has the normalized matching property, which yields that $C[n, k, t]$ has the strong Sperner property and the LYM property.
\end{abstract}

\section{INTRODUCTION}

A finite poset $P$ is graded if every maximal chain of $P$ has the same length, which is called the rank of $P$ denoted by $r(P)$. Thus, for each $x \in P$, every maximal chain with $x$ as the top element has the same length, which is called the rank of the element $x$ and denoted by $r(x)$. Here the length of a chain with $k$ elements is $k-1$. Let $P_{i}$ denote the $i$ th rank of $P$ which consists of all $x \in P$ with $r(x)=i(0 \leq i \leq r(P))$. An antichain is a subset $A \subseteq P$ of which no two elements are comparable in $P$. A $k$-family is a subset of $P$ that contains no chains of length $k$. Clearly, each $P_{i}$ is an antichain, and each union of $k$ ranks of $P$ is a $k$-family.

We say a graded poset $P$ is $k$-Sperner, or has the $k$-Sperner property, if a union of $k$ ranks of $P$ is a maximal-sized $k$-family in $P$. 1-Sperner is just called Sperner. We say $P$ is strong Sperner, or has the strongly Sperner property if it is $k$-Sperner for all $k$. We say $P$ is unimodal if the sequence of the Whitney numbers of $P$ is unimodal, i.e., $W_{0} \leq \ldots \leq W_{l-1} \leq W_{l} \geq W_{l+1} \geq \ldots \geq W_{n}$ for some $l$, where $n=r(P)$ and $W_{i}=\left|P_{i}\right|$. Similarly, $P$ is $\log$ concave if the sequence of Whitney numbers of $P$ is log concave: $W_{m}^{2} \geq W_{m-1} W_{m+1}$ for all $m$. It is clear that $P$ is

Received November 8, 2004, revised April 15, 2005.

Communicated by Xuding Zhu.

2000 Mathematics Subject Classification: 05D05, 06A07.

Key words and phrases: Poset, Subspace lattice, Sperner property, Normalized matching property.

Supported by the National Natural Science Foundation of China (Grant No. 10471016) and the Natural Science Foundation of Liaoning Province of China 
$\log$ concave implies that $P$ is unimodal. For $A \subseteq P_{i}$ where $0 \leq i<r(P)$, define $\nabla(A)=\left\{b \in P_{i+1}: b \geq a\right.$ for some $\left.a \in A\right\}$. We say $P$ has the normalized matching property (NM) or is normal if

$$
\frac{|\nabla(A)|}{|A|} \geq \frac{\left|P_{i+1}\right|}{\left|P_{i}\right|} \quad \text { for all } A \subseteq P_{i}, \quad i=0,1,2, \ldots, r(P)-1 .
$$

We say $P$ has the LYM property, or is a LYM poset if for every antichain $A$ in $P$, the following inequality holds:

$$
\sum_{i=0}^{r(P)} \frac{\left|A \cap P_{i}\right|}{\left|P_{i}\right|} \leq 1 .
$$

It is well known that the NM property and the LYM property are equivalent, and they imply the strong Sperner property [3].

Let $C(n, k)$ be the collection of all subsets of an $n$-set $Y$ which intersect a fixed $k$-subset of $X$. Then $C(n, k)$ is a natural generalization for the subset lattice. Lih [9] first observed this and showed that $C(n, k)$ has the Sperner property. Griggs [2] further showed that $C(n, k)$ has several strong properties.

Let $V_{n}(q)$ denote an $n$-dimensional vector space ( $n$-space, for short) over the finite field with $q$ elements and let $L\left(V_{n}(q)\right)$ be the lattice of subspaces of $V_{n}(q)$, ordered by inclusion. If there is no confusion, write them as $V$ and $L(V)$ for short. Let $K$ be a fixed $k$-dimensional subspace of $V_{n}(q)$ and let $C[n, k, t]$ be the set of the subspaces $S$ such that $\operatorname{dim}(S \cap K) \geq t$ for $1 \leq t \leq k$. Then $C[n, k, t]$ can be regarded as a vector space analogue of $C(n, k)$.

Main results of this paper are contained in the following theorem.

Theorem 1.1. For $1 \leq t \leq k, C[n, k, t]$ has the normalized matching property. Consequently, $C[n, k, t]$ has the strong Sperner property and the LYM property.

We shall expatiate on the way of proving the theorem and present some preliminary results in the next section, and complete the proof of the theorem in Section 3.

\section{Preliminary Results}

Our argument is based on the following theorem which was proved by Kleitman, Edelberg and Lubell in [7]. (See also [1]).

Theorem 2.1. Let $P$ be a poset and $\Gamma$ a permutation group acting on $P$ which preserves the order relation on $P$. Then $P$ contains a maximal sized antichain which is invariant under the action of $\Gamma$. 
Based on the above theorem, the first author proved a conjecture that the subgroup lattice $L_{k^{n}}(p)$ of an abelian $p$-group has the Sperner property in [4], he also gave an NM version of the KEL Theorem, stated as follows.

Theorem 2.1. Let $P$ and $\Gamma$ be the same as in the KEL Theorem. Then for every $i$, there is a subset $A_{i}$ of $P_{i}$ which is invariant under the action of $\Gamma$ and

$$
\frac{\left|\nabla\left(A_{i}\right)\right|}{\left|A_{i}\right|}=\min \left\{\frac{|\nabla(B)|}{|B|}: B \subseteq P_{i}\right\}
$$

As stated in [6], some extremal problems can be considered in a weighted poset $(P, w)$, which is a poset $P$ together with a function (called a weighted function) $w$ from $P$ into the set of non-negative real numbers. The weight $w(A)$ of a subset $A$ of $P$ is defined by $w(A)=\sum_{a \in A} w(a)$. Every poset $P$ can be considered as a weighted poset $(P, w)$, where $w \equiv 1$, that is, $w(x)=1$ for all $x \in P$. A weighted poset $(P, w)$ has the NM property if

$$
\frac{w(\nabla(A))}{w(A)} \geq \frac{w\left(P_{i+1}\right)}{w\left(P_{i}\right)} \text { for all } A \subseteq P_{i}, \quad i=0,1,2, \ldots, r(P)-1 .
$$

Let $\Gamma$ be a permutation acting on $(P, w)$ which preserving the order relation and the weight on $P$, that is, for every $\gamma \in \Gamma, x \leq y$ in $P$ implies $\gamma(x) \leq \gamma(y)$, and $w(\gamma(x))=w(x)$. Then we have the quotient poset $\left(P / \Gamma, w_{\Gamma}\right)$, where $P / \Gamma$ consists of the $\Gamma$-orbits ordered as follows: $\Gamma(x) \leq \Gamma(y)$ in $P / \Gamma$ if $x^{\prime} \leq y^{\prime}$ for some $x^{\prime} \in \Gamma(x)$ and $y^{\prime} \in \Gamma(y)$, and the weight function $w_{\Gamma}$ is given by $w_{\Gamma}(\Gamma x)=|\Gamma x| w(x)$. Then, Theorem 2.2 is restated as follows.

Theorem 2.3. $(P, w)$ has the $N M$ property if and only if $\left(P / \Gamma, w_{\Gamma}\right)$ does.

By this theorem the first author proved that $L_{k^{n}}(p)$ has the NM property for sufficiently large prime number $p$ [5]. In this paper we shall use this theorem to prove Theorem 1.1.

Let $G L(V)$ denote the general linear group over $V$ consisting of all invertible linear transformations of $V$. Let $G L(K \mid V)$ denote the subgroup of $G L(V)$ that fixes $K$. Then the restriction of $G L(K \mid V)$ on $K$ is just the general linear group $G L(K)$. Also, $G L(K \mid V)$ can be induced to the general linear group $G L(V / K)$, where $V / K$ is the quotient space. It is easily seen that $G L(K \mid V)$ induces an order preserving permutation group acting on $C[n, k, t]$.

For any nonnegative integers $i$ and $j$, let $\langle i, j\rangle$ denote the set of all subspaces $A \in L(V)$ such that $\operatorname{dim}(A)=i+j$ and $\operatorname{dim}(A \cap K)=i$. Then if $t \leq i \leq k$, $\langle i, j\rangle$ is an orbit of $G L(K \mid V)$ in $C[n, k, t]$ and the quotient poset is

$$
\bar{C}[n, k, t]=C[n, k, t] / G L(K \mid V)=\{\langle i, j\rangle: t \leq i \leq k, 0 \leq j \leq n-k\} .
$$


The ordering of $\bar{C}[n, k, t]$ is defined as follows: $\langle i, j\rangle \leq\left\langle i^{\prime}, j^{\prime}\right\rangle$ if $i \leq i^{\prime}$ and $j \leq j^{\prime}$. From this it follows that $\bar{C}[n, k, t]$ is isomorphic to the direct product of two chains of lengths $k-t$ and $n-k$, respectively.

Given a $\mu=\langle i, j\rangle \in \bar{C}[n, k, t]$, by $w(\mu)$ we denote the cardinality of the orbit $\mu$. Then, Theorem 2.3 says that $C[n, k, t]$ has the NM property if and only if the weighted lattice $(\bar{C}[n, k, t], w)$ employs the NM property. To express the weight $w(\mu)$ we introduce a few of notations.

Define $\{1\}=1,\{i\}=1+q+\cdots+q^{i-1}=\frac{q^{i}-1}{q-1}$ and $\{i\} !=\{1\}\{2\} \cdots\{i\}$. Then $\left[\begin{array}{c}n \\ m\end{array}\right]:=\frac{\{n\} !}{\{n-m\} !\{m\} !}$, the $q$-binomial coefficient, is known for the number of the $m$-subspaces of $V_{n}(q)$ and have the following recursion:

$$
\left[\begin{array}{c}
n+1 \\
i
\end{array}\right]=\left[\begin{array}{l}
n \\
i
\end{array}\right]+q^{n+1-i}\left[\begin{array}{c}
n \\
i-1
\end{array}\right]
$$

and

$$
\left[\begin{array}{c}
n+1 \\
i
\end{array}\right]=\left[\begin{array}{c}
n \\
i-1
\end{array}\right]+q^{i}\left[\begin{array}{c}
n \\
i
\end{array}\right]
$$

We state two well-known results as two lemmas.

Lemma 2.4. [10]. For any $\langle i, j\rangle \in \bar{C}[n, k, t]$,

$$
w(\langle i, j\rangle)=q^{j(k-i)}\left[\begin{array}{c}
n-k \\
j
\end{array}\right]\left[\begin{array}{c}
k \\
i
\end{array}\right] .
$$

Given $\mu=\langle i, j\rangle \in \bar{C}[n, k, t]_{s}$, we define two order-raising operators $\phi$ and $\varphi$ which map elements of $\bar{C}[n, k, t]_{s}$ into $\bar{C}[n, k, t]_{s+1}$ as follows:

$$
\phi(\langle i, j\rangle)=\langle i+1, j\rangle \text { if } i<k \text {; and } \varphi(\langle i, j\rangle)=\langle i, j+1\rangle \text { if } j<n-k .
$$

It is easy to see that

$$
\nabla(\mu)=\left\{\begin{array}{cc}
\{\phi(\mu), \varphi(\mu)\} & \text { if } i<k \text { and } j<n-k \\
\{\varphi(\mu)\} & \text { if } i=k \text { and } j<n-k \\
\{\phi(\mu)\} & \text { if } i<k \text { and } j=n-k
\end{array}\right.
$$

Now, we introduce another partial order $\preceq$ on $\bar{C}[n, k, t]_{s}$ for $1 \leq s \leq n-1$ :

$$
\langle i, j\rangle \preceq\left\langle i^{\prime}, j^{\prime}\right\rangle \text { iff } j \leq j^{\prime} .
$$

Under this partial order $\bar{C}[n, k, t]_{s}$ is a chain.

Lemma 2.5. Let $\mu=\langle i, j\rangle, \nu=\left\langle i^{\prime}, j^{\prime}\right\rangle \in \bar{C}[n, k, t]_{s}$ and $\mu \preceq \nu$. Then 
(i) $\frac{w(\phi(\mu))}{w(\mu)} \leq \frac{w(\phi(\nu))}{w(\nu)}$ if $i<k$;

(ii) $\frac{w(\varphi(\mu))}{w(\mu)} \geq \frac{w(\varphi(\nu))}{w(\nu)}$ if $j<n-k$.

Proof. If $i<k$, by Lemma 2.4, we have

$$
\frac{w(\phi(\mu))}{w(\mu)}=\frac{q^{j(k-i-1)}\left[\begin{array}{c}
n-k \\
j
\end{array}\right]\left[\begin{array}{c}
k \\
i+1
\end{array}\right]}{q^{j(k-i)}\left[\begin{array}{c}
n-k \\
j
\end{array}\right]\left[\begin{array}{c}
k \\
i
\end{array}\right]}=\frac{\left[\begin{array}{c}
k \\
i+1
\end{array}\right]}{q^{j}\left[\begin{array}{c}
k \\
i
\end{array}\right]}=\frac{\{k-i\}}{q^{j}\{i+1\}}
$$

and

(8) $\frac{w(\varphi(\mu))}{w(\mu)}=\frac{q^{(j+1)(k-i)}\left[\begin{array}{c}n-k \\ j+1\end{array}\right]\left[\begin{array}{l}k \\ i\end{array}\right]}{q^{j(k-i)}\left[\begin{array}{c}n-k \\ j\end{array}\right]\left[\begin{array}{c}k \\ i\end{array}\right]}=\frac{q^{k-i}\left[\begin{array}{c}n-k \\ j+1\end{array}\right]}{\left[\begin{array}{c}n-k \\ j\end{array}\right]}=\frac{q^{k-i}\{n-k-j\}}{\{j+1\}}$.

For convenience, let $i^{\prime}=i-1$ and $j^{\prime}=j+1$. Then by (7) and (8) we obtain that

$$
\frac{w(\phi(\mu))}{w(\mu)} / \frac{w(\phi(\nu))}{w(\nu)}=\frac{\{k-i\}}{q^{j}\{i+1\}} \times \frac{q^{j+1}\{i\}}{\{k-i+1\}}=\frac{q\{k-i\}\{i\}}{\{k-i+1\}\{i+1\}}<1
$$

and

$$
\begin{aligned}
\frac{w(\varphi(\mu))}{w(\mu)} / \frac{w(\varphi(\nu))}{w(\nu)} & =\frac{q^{k-i}\{n-k-j\}}{\{j+1\}} \times \frac{\{j+2\}}{q^{k-i+1}\{n-k-j-1\}} \\
& =\frac{\{n-k-j\}\{j+2\}}{q\{n-k-j-1\}\{j+1\}}>1
\end{aligned}
$$

hold for each $q \geq 1$. The assertions follows by induction.

The following trivial result is used repeatedly in the sequel.

Lemma 2.5. Let $A_{i}, B_{i}>0$ for $1 \leq i \leq k$ and $A_{i} / B_{i} \geq A_{1} / B_{1} \geq \ell$ for $2 \leq i \leq k$. Then

$$
\frac{A_{2}+\cdots+A_{k}}{B_{2}+\cdots+B_{k}} \geq \frac{A_{1}+A_{2}+A_{3}+\cdots+A_{k}}{B_{1}+B_{2}+B_{3}+\cdots+B_{k}} \geq \ell .
$$

\section{Proof of Theorem 1.1}

For convenience, we use $\bar{C}_{s}$ denote the rank $\{\langle i, j\rangle: i+j=s\}$. In order to complete the proof it suffices to prove that for any $1 \leq s \leq n-1$, 


$$
\frac{w(\nabla(M))}{w(M)} \geq \frac{w\left(\bar{C}_{s+1}\right)}{w\left(\bar{C}_{s}\right)}
$$

holds for every $M \subseteq \bar{C}_{s}$.

Suppose $\bar{C}_{s}=\left\{\mu_{1}, \mu_{2}, \ldots, \mu_{r}\right\}$ with $\mu_{1} \preceq \mu_{2} \preceq \cdots \preceq \mu_{r}$. For $1 \leq i \leq r$, define $S_{i}=\left\{\mu_{i}, \mu_{i+1}, \ldots, \mu_{r}\right\}$ and $T_{i}=\left\{\mu_{1}, \mu_{2}, \ldots, \mu_{i}\right\}$. Thus, we immediately have that $w\left(S_{i}\right)$ 's decrease monotonic with $i$ and $w\left(T_{i}\right)$ 's increase monotonic with $i$.

Lemma 3.1. With the notations as above we have

(i) $\frac{w\left(\nabla\left(S_{r}\right)\right)}{w\left(S_{r}\right)} \geq \frac{w\left(\nabla\left(S_{r-1}\right)\right)}{w\left(S_{r-1}\right)} \geq \cdots \geq \frac{w\left(\nabla\left(S_{1}\right)\right)}{w\left(S_{1}\right)}=\frac{w\left(\bar{C}_{s+1}\right)}{w\left(\overline{C_{s}}\right)}$.

(ii) $\frac{w\left(\nabla\left(T_{1}\right)\right)}{w\left(T_{1}\right)} \geq \frac{w\left(\nabla\left(T_{2}\right)\right)}{w\left(T_{2}\right)} \geq \cdots \geq \frac{w\left(\nabla\left(T_{r}\right)\right)}{w\left(T_{r}\right)}=\frac{w\left(\bar{C}_{s+1}\right)}{w\left(\bar{C}_{s}\right)}$.

Proof. By definition we have that

$$
\mu_{1}=\left\{\begin{array}{cc}
\langle k, s-k\rangle & \text { if } s \geq k \\
\langle s, 0\rangle & \text { if } s<k
\end{array}\right.
$$

and

$$
\mu_{r}=\left\{\begin{array}{cc}
\langle t, s-t)\rangle & \text { if } s-t<n-k \\
\langle s-(n-k), n-k\rangle & \text { if } s-t \geq n-k
\end{array}\right.
$$

It is easy to see that

$$
\nabla\left(S_{1}\right)= \begin{cases}\nabla\left(S_{2}\right) & \text { if } s \geq k \\ \phi\left(\mu_{1}\right) \cup \nabla\left(S_{2}\right) & \text { if } s<k\end{cases}
$$

and for $i \geq 2$,

$$
\nabla\left(S_{i}\right)= \begin{cases}\left\{\phi\left(\mu_{i}\right), \phi\left(\mu_{i+1}\right), \ldots, \phi\left(\mu_{r}\right), \varphi\left(\mu_{r}\right)\right\} & \text { if } s-t<n-k \\ \left\{\phi\left(\mu_{i}\right), \phi\left(\mu_{i+1}\right), \ldots, \phi\left(\mu_{r}\right)\right\} & \text { if } s-t \geq n-k .\end{cases}
$$

We thus obtain by Lemma 2.5 and Lemma 2.6 that

$$
\frac{w\left(\nabla\left(S_{i}\right)\right)}{w\left(S_{i}\right)}=\frac{w\left(\phi\left(\mu_{i}\right)\right)+w\left(\nabla\left(S_{i+1}\right)\right)}{w\left(\mu_{i}\right)+w\left(S_{i+1}\right)} \leq \frac{w\left(\nabla\left(S_{i+1}\right)\right)}{w\left(S_{i+1}\right)}
$$

hold for $i>1$ or $i=1$ while $s<k$. If $i=1$ and $s \geq k$, then $S_{1}=\left\{\mu_{1}\right\} \cup S_{2}$ and $\nabla\left(S_{1}\right)=\nabla\left(S_{2}\right)$, so

$$
\frac{w\left(\nabla\left(S_{1}\right)\right)}{w\left(S_{1}\right)}=\frac{w\left(\nabla\left(S_{2}\right)\right)}{w\left(\mu_{1}\right)+w\left(S_{2}\right)}<\frac{w\left(\nabla\left(S_{2}\right)\right)}{w\left(S_{2}\right)} .
$$


The assertion (1) follows.

Similarly, for $i \leq r$, we have

$$
\nabla\left(T_{i}\right)=\left\{\begin{aligned}
\left\{\phi\left(\mu_{1}\right), \varphi\left(\mu_{1}\right), \varphi\left(\mu_{2}\right), \ldots, \varphi\left(\mu_{i}\right)\right\} & \text { if } s<k \\
\left\{\varphi\left(\mu_{1}\right), \varphi\left(\mu_{2}\right), \ldots, \varphi\left(\mu_{i}\right)\right\} & \text { if } s \geq k .
\end{aligned}\right.
$$

Then (2) follows from Lemma 2.5 and Lemma 2.6.

Lemma 3.2. With the notations as in the above lemma let $M=\left\{\mu_{i}, \mu_{i+1}, \ldots\right.$, $\left.\mu_{j}\right\}$ be a subset of $\bar{C}_{s}$, where $1 \leq i \leq j \leq r$. Then $\frac{w(\nabla(M))}{w(M)} \geq \frac{w\left(\bar{C}_{s+1}\right)}{w\left(\bar{C}_{s}\right)}$.

Proof. By definition we have that $M=S_{i} \cap T_{j}$ and $\nabla(M)=\nabla\left(S_{i}\right) \cap \nabla\left(T_{j}\right)$. It is easy to see that $S_{i} \cup T_{j}=\bar{C}_{s}$ and $\nabla\left(S_{i}\right) \cup \nabla\left(T_{j}\right)=\bar{C}_{s+1}$, which implies that $w(M)=w\left(S_{i}\right)+w\left(T_{j}\right)-w\left(\bar{C}_{s}\right)$ and $w(\nabla(M))=w\left(\nabla\left(S_{i}\right)\right)+w\left(\nabla\left(T_{j}\right)\right)-$ $w\left(\bar{C}_{s+1}\right)$. We thus obtain that

$$
\frac{w(\nabla(M))}{w(M)}=\frac{w\left(\nabla\left(S_{i}\right)\right)+w\left(\nabla\left(T_{j}\right)\right)-w\left(\bar{C}_{s+1}\right)}{w\left(S_{i}\right)+w\left(T_{j}\right)-w\left(\bar{C}_{s}\right)} \geq \frac{w\left(\bar{C}_{s+1}\right)}{w\left(\bar{C}_{s}\right)} .
$$

The last equality follows from Lemma 3.1.

Proof of Theorem 1.1 Given a subset $M$ of $\bar{C}_{s}$, set $M=M_{1} \cup \cdots \cup M_{\ell}$ $(\ell \geq 1)$, where $M_{k}=\left\{\mu_{i_{k}}, \mu_{i_{k}+1}, \ldots, \mu_{j_{k}}\right\}(k=1,2, \ldots, \ell), 1 \leq i_{1} \leq j_{1}<$ $i_{2} \leq j_{2}<\cdots<i_{\ell} \leq j_{\ell} \leq r$. Then $M_{i} \cap M_{j}=\emptyset$ and $\nabla\left(M_{i}\right) \cap \nabla\left(M_{j}\right)=\emptyset$ for $i \neq j$. We thus obtain that $w(M)=w\left(M_{1}\right)+\cdots+w\left(M_{\ell}\right)$ and $w(\nabla(M))=$ $w\left(\nabla\left(M_{1}\right)\right)+\cdots+w\left(\nabla\left(M_{\ell}\right)\right)$. Then the inequality $\frac{w(\nabla(M))}{w(M)} \geq \frac{w\left(\bar{C}_{s+1}\right)}{w\left(\bar{C}_{s}\right)}$ follows from Lemma 3.2 and Lemma 2.6.

Remark. Note that all inequalities above hold for $q \geq 1$. By putting $q=1$ in Theorem 1.1 we reobtain that $C(n, k, t)$ has the normalized matching property, which has been proved by Griggs in [2].

\section{REFERENCES}

1. R. Freese, An application of Dilworth's lattice of maximal antichains, Discrete Math., 7 (1974), 107-109.

2. J. R. Griggs, Collections of subsets with the sperner property, Trans. Amer. Math. Soc., 269 (1982), 575-591.

3. J. R. Griggs, On chains and Sperner $k$-families in ranked posets, J. Combin. Theory Ser. A, 28 (1980), 156-168. 
4. J. Wang, Proof of a conjecture on the sperner property of the subgroup lattice of an abelian p-group, Ann. of Combin., 2 (1998), 85-101.

5. J. Wang, Normalized matching property of the subgroup lattice of an abelian $p$-group, Discrete Math., 257 (2002), 559-574.

6. K. Engel, Sperner theory, Cambridge University Press, Cambridge, 1997. P. 160.

7. D. Kleitman, M. Edelberg and D. Lubel, Maximal sized antichains in partial orders, Discrete Math., 1 (1971), 47-53.

8. D. Kleitman, On an extremal property of antichains in partial orders. The LYM property and some of its implications and application, in: Combinatorics, Part 2, Math. Center Tracts, \& Math. Centrum Amsterdam, 56 1974, 77-90.

9. K. W. Lih, Sperner families over a subset, J.Combin. Theory Ser. A, 29 (1980), $182-185$.

10. Y. Wang, On a class of subspace lattice, J. Math. Res. Exp., 19 (1999), 341-348.

\author{
Jun Wang ${ }^{1,2}$ and Huajun Zhang $^{1} *$ \\ ${ }^{1}$ Department of Applied Mathematics, \\ Dalian University of Technology, \\ Dalian 116024, P. R. China \\ E-mail: zhhj1979@yahoo.com.cn \\ ${ }^{2}$ College of Advanced Science and Technology, \\ Dalian University of Technology, \\ Dalian 116024, P. R. China \\ E-mail: junwang@dlut.edu.cn
}

